\title{
Behandlungseinheiten
}

\section{Einfach zu bedienen. Einfach nachzurüsten. Einfach zuverlässig.}

Mit der neuen ESTETICA E50 Life spüren Sie die Unterstützung einer Behandlungseinheit, die Ihre Arbeitsabläufe in der täglichen Praxis optimal begleitet. Ohne zeitaufwändiges Einarbeiten und lästiges Suchen führen Sie die Direkttasten schnell und intuitiv. Das Arztelement der Einheit bietet Ihnen in Positionierung und Instrumentierung die Flexibilität: im Sitzen oder Stehen, alleine oder mit Assistenz. Mit dem flexiblen Wendepolster und der 2-Gelenk-Kopfstütze lässt sich für jeden Patienten die richtige Liegeposition finden. Das Arztelement, als Tisch- oder Schwingenversion, die Trayablage und das
Assistenzelement lassen sich durch den großen Bewegungsradius optimal positionieren. Die neue Systemsoftware CONEXIO zeigt Ihnen über die benutzerfreundliche Bedienfolie der Einheit den ausgewählten Patienten direkt an. Steuern Sie Ihre Patientenkommunikation jetzt vom Arztelement aus. Nutzen Sie zur Aufklärung Ihrer Patienten hochauflösende Aufnahmen und Bilder in hoher Tiefenschärfe und Farbwiedergabe. Speziell hierfür wurden die neue, leicht $\mathrm{zu}$ bedienende KaVo Intraoralkamera ERGOcam One und die neuen HD Flatscreens entwickelt. Die KaVo ESTETICA E50 Life ist eine saubere

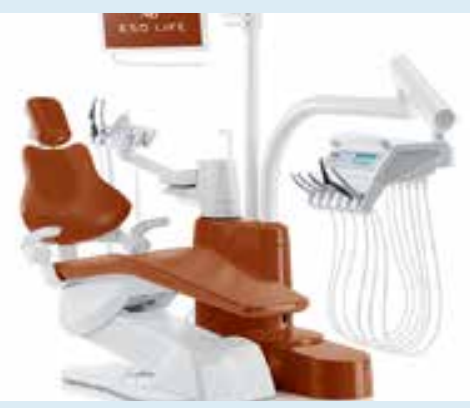

Sache, wichtige Bestandteile lassen sich zum Reinigen und Desinfizieren einfach abnehmen. Starten Sie einfach das automatisierte HYDROclean-Programm zur Reinigung der Amalgamabscheide-, Saugund Abflusssysteme. Zusätzlich sorgt die Dauerentkeimung mit KaVo OXYGENAL 6 für die permanente Reduktion von Bakterien und Keimen.

Nach einer Pressemitteilung der

KaVo Dental GmbH, Biberach / Riss 